\title{
Reproductive health of female childhood cancer survivors
}

\author{
Rūta Žulpaitè ${ }^{1}$, Žana Bumbuliené ${ }^{2}$ \\ ${ }^{1}$ Vilnius University Faculty of Medicine, Vilnius, Lithuania \\ ${ }^{2}$ Vilnius University Faculty of Medicine, Institute of Clinical Medicine, Clinic of Obstetrics and Gynaecology, Vilnius, Lithuania
}

\begin{abstract}
Current treatment schemes of childhood cancer are usually effective enough to enable successful management of the disease. With the high rates of survival, another problem arises because patients often suffer much later from side effects of the toxic therapy. A common complication caused by cancer treatment is impairment of the female reproductive system including dysfunction of the hypothalamus and hypophysis, the killing of gonadal cells, and uterine injury. This may lead to altered pubertal timing, gonadotropin insufficiency or deficiency, acute ovarian failure, premature ovarian insufficiency, sexual dysfunction, and complicated pregnancy. The severity of these side effects depends a lot on the patient's age at treatment and the particularities of their chemo- and/or radiotherapy regimens.

While some types of cancer require aggressive treatment, and therefore negative side effects cannot be avoided, strategies which preserve the patient's reproductive potential are essential. Such strategies are more established in the treatment of adult women, however there are also promising opportunities in the treatment of pediatric oncology patients. Ovarian transposition is already widely applied before pelvic radiotherapy. Cryopreservation of ovarian tissue, cryopreservation and in vitro maturation of immature oocytes, or cryopreservation of mature oocytes when the patient's age is appropriate, have also shown to have promising results in pediatric patients. Concurrent combinations of several techniques can also be successful.

Counselling of pediatric patients and their families is challenging, and the urgent commencement of anticancer therapies often discourages attempts to preserve the girl's reproductive system. Given that successful methods of fertility preservation are already accessible, it is crucial not to leave this topic aside at the time of diagnosis.
\end{abstract}

Key words: childhood cancer, reproductive health, fertility preservation

Ginekologia Polska 2018; 89, 5: 280-286

\section{INTRODUCTION}

Each year about 15,000 children ( $0-15$ years), 20,000 adolescents and young adults (15-24 years) in Europe hear the diagnosis of cancer. Fortunately, current treatment schemes are effective enough to enable successful management of the disease, therefore survival of these patients at 5 years after diagnosis is about $80 \%$ [1].

Considering outcomes, three groups of childhood cancers can be distinguished: good, poor and very poor prognosis. The first group has higher than $85 \%$ survival rate after 5 years from diagnosis and includes acute lymphoblastic leukaemia, lymphomas, retinoblastoma, renal tumours. To manage these diseases multidisciplinary schemes including intensive modes of cytotoxic drugs are usually used. Acute myeloid leukaemia, some central nervous system (CNS) tumours, bone and soft tissue sarcomas, neuroblastoma have poor prognosis with a 5 -year survival less than $50 \%$. Diffuse intrinsic pontine glioma, high-risk neuroblastoma and metastatic sarcomas have extremely poor prognosis [2].

Overall, many childhood cancer survivors have all the life ahead. However, they may suffer late side effects of treatment, which significantly impair the quality of life. Among other organs and tissues, reproductive system is usually affected which may cause not only metabolic, immunological or growth impairment but also a psychosocial disfunction. Even knowing about the risk of possibile infertility extremely

Corresponding author:

Rūta Žulpaitè

Vilnius University Faculty of Medicine, M. K. Čiurlionio St. 21, LT-03101, Vilnius, Lithuania

e-mail: ruta.zulp@gmail.com 
Table 1. Risk of subfertility after treatment of different types of childhood cancers [7]

\begin{tabular}{|l|l|l}
\hline Low risk $(<\mathbf{2 0 \% )}$ & Medium risk & High risk $(>\mathbf{8 0} \%)$ \\
\hline - Acute lymphoblastic leukaemia & - Acute myeloblastic leukaemia & - Whole-body irradiation \\
- Wilms' tumour & - Hepatoblastoma & - Localised pelvic radiotherapy \\
- Soft tissue sarcoma: stage I & - Osteosarcoma & - Chemotherapy conditioning for bone- \\
- Germ-cell tumours (with gonadal & - Ewing's sarcoma: non-metastatic & - Hodgkin's disease: treatment with \\
preservation and no radiotherapy) & - Soft tissue sarcoma: stage II or III & alkylating-drugs \\
- Retinoblastoma & - Neuroblastoma & - Soft-tissue sarcoma: stage IV (metastatic) \\
- Brain tumour: surgery only, cranial & - Non-Hodgkin lymphoma & - Hodgin's lymphoma: alternating treatment \\
irradiation $<24$ Gy & - Erain tumour: craniospinal radiotherapy, & \\
& cranial irradiation $>24$ Gy &
\end{tabular}

affect well-being and intimate relationships of adolescent or young adult childhood cancer survivors [3]. This reveals a crucial importance to preserve a good state of the reproductive health in every pediatric oncologic patient.

\section{MECHANISMS OF REPRODUCTIVE SYSTEM IMPAIRMENT}

Reproductive late effects in female survivors of childhood cancer include altered pubertal timing (precocious, early, rapid tempo, delayed), gonadotropin insufficiency or deficiency, acute ovarian failure (AOF), premature ovarian insufficiecy (POI), uterine growth impairment and/or vascular injury, vaginal fibrosis or stenosis, sexual dysfunction, dyspareunia, complicated pregnancy [4].

All women have a non-renewable pool of follicles from birth, thus the potential of fertility is limited by available oocytes. A peak in primordial follicles number (6-7 million) present in 20-weeks female fetus. Following the progresive atresia, the quantity of oocytes decline to 1-2 million at birth and 400,000-600,000 at menarche, leading to menopause with less than 1000 remaining follicles [5].

The pool of primordial follicles may be substantially diminished by cancer treatment through its associated cell kill [6] leading to AOF or POI. AOF is a loss of ovarian function within 5 years after diagnosis and manifest as amenorrhea or lack of pubertal development in a prepubertal girl. POI means termination of menses before the age of 40 , following years of a normal ovarian function ("fertile window") [4]. The number of oocytes decline with the age according to normal physiology, therefore the older the ovary, the higer the risk of acute failure. On the contrary, younger age at treatment seems to be a partially protective factor due to higher number of primordial follicles at the time of toxic agents exposition [7]. Childhood Cancer Survivor Study (CCSS) revealed that AOF appears in a relatively small number of childhood cancer survivors (6,3\%) [8]. More commonly, young women after cancer treatment present with $\mathrm{POI}$ - this occurs much more often in childhood cancer survivors than in control group ( $8 \%$ vs. $0,8 \%$ ) [9]. Postmenopausal levels of follicle-stimulating hormone (FSH) (as defined by the measuring laboratory) in the background of irregular menses before age 40 years is a signal of increased speed of follicles atresia and ovarian failure. Generally the risk of subfertility can be classified according to the type of cancer and its associated treatment (Tab. 1).

\section{Impact of radiotherapy}

Brain tumours, such as meduloblastoma, astrocytoma, poorly differenciated neuroectodermal tumours and others, in addition to surgery usually require cranial irradiation. The injury of hypothalamus and hypophysis leads to endocrine dysfunction impairing pubertal development, menstrual regulation and reproductive function. It is known that immature hypothalamus is more radiosensitive than postpubertal one [10]. Precocious puberty is the most common consequence in girls following 18-24 Gy radiation. Such doses activate hypothalamus which secretes gonadotropin-releasing hormone $(\mathrm{GnRH})$ in a pulsatile way, thus influencing hypophysis to produce gonadotropins and stimulate ovaries [11]. In contrast, 40-50 Gy or even 30 Gy doses of radiation might directly injur hypothalamus-pituitary-gonadal axis. This exposition before or during pubertal development more commonly cause central hypogonadism which presents as a pubertal delay and interruption of maturation with oligomenorrhoea or amenorrhoea. Doses of $40 \mathrm{~Gy}$ or higher lead to hyperprolactinaemia, which can also cause amenorrhoea [10-12].

Pelvic and abdominal irradiation directly affects ovaries and uterus. Pediatric tumours, such as pelvis Ewing sarcoma, rhabdomyosarcoma of the bladder, vagina or uterus, soft tissue sarcomas are managed with pelvic radiotherapy. Wallace et al. using Faddy-Gosden mathematical model investigated that the dose able to destroy half of the immature oocytes (LD50) is less than 2 Gy [13]. Irradiation induces apoptosis and causes direct mitochondrial deoxyribonucleic acid (DNA) lesions in oocytes, damages blood vessels, impairs neovascularization and causes focal fibrosis in ovaries [14]. These mechanisms accelerate physiological follicular pool depletion and impair ovarian endocrine function. Wallace et al. also found that the effective sterilizing dose (ESD), or dose of fractionated radiotherapy at which ovarian failure 
occurs immediately after treatment in $97.5 \%$ of patients, decreases with increasing age at treatment. ESD at birth was estimated to be $20.3 \mathrm{~Gy}$; at 10 years $18.4 \mathrm{~Gy}$; at 20 years $16.5 \mathrm{~Gy}$; at 30 years $14.3 \mathrm{~Gy}$ [13]. Some patients even with normal menstrual cycle after radiotherapy may present with a decreased concentration of anti-Müllerian hormone (AMH). Since AMH is an accurate index of ovarian reserve, these women have a high risk of POI [15-16]. There is no sufficient technique to estimate the duration of „fertile window", therefore it is essential to predict and early detect a compromised ovarian function. Anticipation of shortened reproductive time may be helpful for family planning and expecting the necessity for hormone therapy in the future.

Pelvic irradiation in childhood negatively affect uterus, increasing risk of spontaneous miscarriages, preterm labor, low-birth-weight infants, placental abnormalities [17]. These pregnancy-related complications are related with reduced uterine volume, damage of uterine vessels, myometrial fibrosis, endometrial injury. Insufficient vascularization reduce uterine response to cytotrophoblast invasion and decrease fetoplacental circulation of blood, thus fetal growth is impaired. Injury of endometrium worsen decidualization process, therefore placental attachment disorders such as placenta accreta or placenta percreta may occur. It is even speculated that radiotherapy may cause diffuse thinning of the myometrium and increase risk of uterine rupture during pregnancy. Prepubertal uterus is more sensitive to pelvic irradiation than adult uterus and doses of approximately 14 to 30 Gy may lead to irreversible uterine dysfunction [18]. In younger than 6-year-old patients uterus is injured much more severe than ovaries [19].

\section{Impact of chemotherapy}

Chemotherapeutic agents have been classified into three risk categories, according to their gonadal toxicity (Tab. 2).

The alkylating agents create covalent junctions between DNA chains, interfere with cleavage during DNA replication and cause disruption of cell division, thus directly injuring oocytes [20] whereas other drugs, such as docetaxel, destroy ovarian-somatic cells with secondary oocyte death [21]. Reproductive outcomes also depend on treatment regime, cumulative dose, age at treatment and ovarian reserve $[4,6]$.
Even less gonadotoxic agents may reduce follicles pool or induce AOF if used in combinations.

Due to the different protocols of chemotherpeutic treatment an oncological disease itself might be a predictor of ovarian injury. Hodgkin lymphoma is considered as a risk factor of ovarian failure with the rate reaching 50\% [20]. The systematic review, including 45 studies, revealed that median age at menopause is $\mathbf{3 3 . 5}$ years in Hodgin lymphoma survivors. The highest prevalence of amenorrhoea was reported in the group of patients exposed to MVPP (chlormethamine, vinblastine, procarbazine and prednisone) $-39-79 \%$. Amenorrhoea rate in Hodgin lymphoma survivors treated with ABVD (adriamycin, bleomycin, vinblastine, dacarbazine) protocol seems to be not higher than the control population (0-3,8\%). After MOPP (chlormethamine, oncovin, procarbazine and prednisone) chemotherapy, amenorrhoea rates of $25-79 \%$ were reported [6]. In contrast, pediatric acute myeloid leukemia has a relatively low risk of ovarian failure and infertility as treatment protocols used for this disease include anthracyclines and citrabine but not alkylating agents. Most acute myeloid leukemia survivors in the study of Molgaard-Hansen et al. had normal pubertal development and fertility, however $13 \%$ of postpubertal females presented with decreased AMH levels [22]. Only a 10\% premature ovarian failure incidence have been reported after chemotherapeutic treatment for acute lymphoblastic leukaemia in prepubertal patients, while high rates of ovarian insufficiency were reported among adolescents and adults with this type of cancer [20].

Bone marrow transplantation is undoubtedly associated with the highest risk of ovarian failure and impaired pubertal development, while high-doses of cyclophosphamide with or without busulfane are used prior to transplantation. Only less than $5 \%$ of such patients report childbearing after treatment [23-24]. Fertility is less affected in patients who undergo transplant as young adults (15-30 years) and get only cyclophosphamide based conditioning regimens [23]. Although conditioning regimens used for children are mainly myeloablative (87\%), the proportion of pediatric patients receiving reduced-intensity/toxicity conditioning regimens has increased from $8 \%$ in 2000 to $16 \%$ in 2015 [24]. Risk-adapted chemotherapy combinations reducing cumulative dose of alkylating agents and including less gonadotoxic

Table 2. Risk categories of various chemotherapeutic drugs according to their gonadotoxicity [20]

High risk

Medium risk

Low risk
Alkylating agents (cyclophosphamide, busulphan, chlorambucil, procarbazine, melphalan, ifosfamide, chlormethamine)

Platinum agents (cisplatin, carboplatin); anthracycline antibiotics (adriamycin [doxorubicin]); taxoids (docetaxel and paclitaxel)

Vinca plant alkaloids (vincristine and vinblastine); anthracycline antibiotics (bleomycin), antimetabolites (methotrexate, 5-fluorouracil, 6-MP [mercaptopurine]) 
drugs are more and more widely used taking into consideration future outcomes. Unfortunatelly, an agressive and gonatotoxic treatment still cannot be avoided in patients with advanced cancers with poor prognosis.

\section{FERTILITY PRESERVATION Ovarian transposition (oophoropexy)}

Rhabdomyosarcomas of the bladder, vagina and uterus, pelvic soft tissue or bone sarcomas such as Ewing's sarcoma, which require radiotherapy implicating pelvis, are the most common indications for oophoropexy. The position of ovaries is decided by surgeon and radiotherapist according to the radiotherapy plan. Ovarian transposition is often performed after neoadjuvant chemotherapy to indicate chemosensivity of the tumour and control the disease [19, 25]. Ovaries can be transposed via laparotomy together with surgical removal of the tumour or via laparascopy, which is the best choice when surgery is not indicated. The gonads are sutured to the peritoneum and marked with metallic clips in order to be detected in radiographs. After cure from cancer, ovaries can be surgically moved into their previous position to allow spontaneous pregnancy in the future [26]. The complications of ovarian transposition include ovarian torsion, pelvic adhesions leading to tubal or bowel obstruction, severe dyspareunia after retrouterine transposition, ovary displacement which can impair fertility and future assisted reproductive techniques. Moreover, radiation scattering and unavoidable movements of ovaries, reduce the reliability of this method. Even after transposition, gonads should be kept away from the radiation field with approximately $2 \mathrm{~cm}$ of organ at risk volume margins [27]. Oophoropexy should be performed as close to the beginning radiotherapy as possible, due to the risk of ovarian remigration.

\section{Gonadotropin releasing hormone analogues (GnRHa)}

Theoretically, GnRHa create pseudoprepubertal conditions by inhibiting hypothalamic-pituitary-ovarian axis, thus making ovaries suppressed and less sensitive to gondatoxic agents. A direct antiapoptotic effect on ovarian germline stem cells has also been noticed [28]. However, GnRHa cannot be applied to pre-pubertal girls while their follicles are immature and ovarian cycle is not settled. Post-pubertal patients may benefit on this method, however there is lack of studies revealing the impact on ovarian suppression for adolescents. While not enough evidence of efficacy and possible harm is currently available, this method is not routinely recommended for pediatric patients [25].

\section{Embryo cryopreservation}

It is a worldwide routinely used technique with satisfactory number of pregnancies. However, approximately
2-3 weeks are needed for ovarian stimulation and such treatment delay is not always acceptable. Most importantly, since sperm is necessary for the creation of embryos, this method is only applicable for adults.

\section{Cryopreservation of mature oocytes}

This method is well-developed and no longer considered experimental [29-30]. Currently, more flexible random-start protocols are available for ovarian stimulation. Therefore in most cases there is no need to wait for a certain day of the menstrual cycle and the delay of treatment initiation is lesser to compare with older methods. Current vitrification techniques allow perfect oocyte survival and following normal development. Noyes et al. in 2009 reported 900 newborns after oocyte cryopreservation [31]. Rate of congenital anomalies was not higher than in normally conceived babies population. Disadvantages of this method include potential side effects of the hormonal interventions, necessity of endovaginal access to retrive oocytes, unavailability for pre-pubertal patients $[25,30]$.

\section{In vitro maturation (IVM) of immature oocytes}

This strategy has been used mostly in adult women in addition to mature oocytes retrieval, to maximize the number of cells suitable for in vitro fertilization (IVF), as well as for the patients who cannot undergo a stymulated cycle, have policystic ovaries syndrome or need immediate cancer treatment. Although multiple pregnancies have been achieved with this technique and results are improving, the overall success rate remains lower than in conventional IVF. IVM can be considered as an additional technique to ovarian tissue cryopreservation and autotransplantation. However, further investigation on this topic is necessary. Currently IVM is considered experimental, especially for children [26]

\section{Ovarian tissue cryopreservation}

A small portion of ovarian cortical tissue or the whole ovary can be cryopreserved and transplanted later or used to retrieve immature oocytes for in vitro maturation [29]. Sexual maturity is not necessary for the procedure, therefore, although considered experimental, this method is currently the only possible option for fertility preservation in prepubertal patients. Ovarian tissue cryopreservation may show good results when offered to the patients with the highest risk of POI but likely to survive. For this evaluation Wallace et al. suggested to use Edinburgh selection criteria (Tab. 3).

At least 80 pregnancies have been reported by far after autotransplantation of cryopreserved adult ovarian tissue with pregnancy rate $23-37 \%$ [33]. Demeestere et al. in 2015 reported the first live birth after autotransplantation of ovarian tissue which was obtained from adolescent girl before the onset of menarche [34]. Return of hormonal 
Table 3. Edinburgh criteria for selection for ovarian tissue cryopreservation [32]

Age $<35$ years

- No previous chemotherapy or radiotherapy (patients aged $<15$ years with previous low-risk chemotherapy should be considered)

- Realistic chance of surviving for at least 5 years

- High risk of treatment-induced immediate ovarian failure (estimated $>50 \%$ )

- Informed consent from patient or (in the case of an incompetent child) from parents

Negative HIV*, syphilis, hepatitis serology

Not pregnant, no existing children

*Human immunodeficiency virus

function after ovarian tissue autotransplantation allow to achieve puberty induction and onset of menses [35]. Ovarian transposition and ovarian tissue cryopreservation are sometimes combined [27, 36]. Autograft can be stored in liquid nitrogen at $-196{ }^{\circ} \mathrm{C}$ for more than ten years [36]. Later the tissue can be returned into pelvis and fixed to the remaining ovary, peritoneum of ovarian fossa or broad ligament, theoretically allowing natural conception. However, ortotopic transplantation is a highly invasive laparoscopic or even laparotomic procedure [26, 29, 33, 36], which may be complicated due to pelvic adhaesions after radiotherapy. Heterotopic transplantation to, e.g. forearm, abdominal wall or chest wall, is technically easier, allows better access for oocyte retrieval and follicular monitoring. Studies showed good results with restored ovarian function and follicular development [37-38]. The first clinical pregnancy and delivery of twins following heterotopic grafting of cryopreserved ovarian tissue has been reported in 2013 [38]. However, IVF is unavoidable to achieve pregnancy in these cases. Vability of autografts can be impaired due to poorer neovascularization in heterothopic position $[26,34,36]$. A theoretical possibility of cancer cells reintroduction with ovarian tissue autograft must be considered, especially for the patients with leukaemia [39]. Although so far any reports of cancer recurrence have been published, laboratory tests should be performed to exclude possible malignancy [39]. Some research with artificial ovary created of three-dimensional (3D) alginate matrigel matrix and filled with patient's follicles has shown satisfactory results in animals. This promising technique would allow to avoid risk of reimplanting malignant cells [26].

\section{COUNSELLING}

Effects on girl's pubertal development and future fertility should be discussed before the treatment plan is confirmed. Multidisciplinary team including oncology and reproductive medicine services or even mental health specialists is essential to achieve satisfactory results. American Society
Table 4. Points for a discussion of infertility and fertility preservation with a patient (or parents or guardians) according to ASCO Practise Guideline [39]

- Inform patient of individual risk

- Discuss common concerns:

- Options of fertility preservation

- Risk of delaying treatment

- Costs necessary for fertility preservation procedures

- Pregnancy risks after cancer

- Refer to appropriate specialists (e.g. reproductive specialists,

mental health professionals, etc.)

of Clinical Oncology (ASCO) recommends clear points for a discussion of infertility and fertility preservation with a patient, parents or guardians (Tab. 4).

Established methods of fertility preservation for pediatric patients should be innitally proposed. Information about currently investigational methods or available experimental protocols should be also provided, especially in cases, when convenional techniques are not appropriate [39]. However, a PanCare project survey revealed that counselling of such patients is not established enough: from 198 European institutions which provided haemato-oncology treatment to children, only 68 institutions responded to questionaire and pretreatment fertility counselling was offered by 64 institutions. Only one third of them worked by a team while patients in other institutions were counselled only by oncohemathologist [40]. Another study showed similar results: in 38 centers which responded to their survey $39 \%$ of patients were councelled and only $29 \%$ received fertility preservation programs [41].

The time necessarry for fertility preservation procedures have to be balanced with the beginning of potentially life-saving anticancer therapies, leading to extreme family stress. Given their preference on intiating cancer management, pediatric oncology providers might not pay enough attention on reproductive issues, thus discouraging these families on attempts to preserve fertility before treatment. Similarly, reproductive specialists may lack competence with aspects of particular oncologic diagnosis. A majority of fertility preservation techniques are considered experimental in children, therefore no guarantees of success can be given to the patient's family. To solve the current problems, PanCare LIFE project including experts form various European and non-European countries is currenly working on universal and cohesive guidelines for fertility issues of pediatric patients facing gonadotoxic therapies [42].

\section{CONCLUSIONS}

Considering high survival rates of pediatric cancer and risk of reproductive health impairment, it is crucial not to leave this topic aside at the time of oncologic diagnosis. 
Several current methods of fertility preservation have already showed good results while some of them are still being developed. Further studies on reducing gonadotoxicity of cancer treatments and preserving reproductive function are necessary to improve counselling and management of these patients, including all the aspects of the disease.

\section{REFERENCES}

1. Gatta G, Botta L, Rossi S, et al. EUROCARE Working Group. Childhood cancer survival in Europe 1999-2007: results of EUROCARE-5--a population-based study. Lancet Oncol. 2014; 15(1):35-47, doi: 10.1016/S14702045(13)70548-5, indexed in Pubmed: 24314616.

2. Ferlay J, Steliarova-Foucher E, Lortet-Tieulent J, et al. Cancer incidence and mortality patterns in Europe: estimates for 40 countries in 2012. Eur J Cancer. 2013; 49(6): 1374-1403, doi: 10.1016/j.ejca.2012.12.027, indexed in Pubmed: 23485231.

3. Nilsson J, Jervaeus A, Lampic C, et al. 'Will I be able to have a baby?' Results from online focus group discussions with childhood cancer survivors in Sweden. Hum Reprod. 2014; 29(12): 2704-2711, doi: 10.1093/humrep/deu280, indexed in Pubmed: 25344069.

4. Hudson MM. Reproductive outcomes for survivors of childhood cancer. Obstet Gynecol. 2010; 116(5): 1171-1183, doi: 10.1097/AOG.0b013e3181f87c4b, indexed in Pubmed: 20966703.

5. Lambalk $C B$, van Disseldorp J, de Koning $\mathrm{CH}$, et al. Testing ovarian reserve to predict age at menopause. Maturitas. 2009; 63(4): 280-291, doi: 10.1016/j.maturitas.2009.06.007, indexed in Pubmed: 19631481.

6. Overbeek A, van den Berg MH, van Leeuwen FE, et al. Chemotherapy-related late adverse effects on ovarian function in female survivors of childhood and young adult cancer: A systematic review. Cancer Treat Rev. 2017; 53: 10-24, doi: 10.1016/j.ctrv.2016.11.006, indexed in Pubmed: 28056411.

7. Wallace $W H$, Anderson RA, Irvine DS. Fertility preservation for young patients with cancer: who is at risk and what can be offered? Lancet Oncol. 2005; 6(4): 209-218, doi: 10.1016/S1470-2045(05)70092-9, indexed in Pubmed: 15811616.

8. Chemaitilly W, Mertens AC, Mitby P, et al. Acute ovarian failure in the childhood cancer survivor study. J Clin Endocrinol Metab. 2006; 91(5): 1723-1728, doi: 10.1210/jc.2006-0020, indexed in Pubmed: 16492690.

9. Green DM, Sklar CA, Boice JD, et al. Ovarian failure and reproductive outcomes after childhood cancer treatment: results from the Childhood Cancer Survivor Study. J Clin Oncol. 2009; 27(14): 2374-2381, doi: 10.1200/JCO.2008.21.1839, indexed in Pubmed: 19364956.

10. Follin C, Erfurth EM. Long-Term Effect of Cranial Radiotherapy on Pituitary-Hypothalamus Area in Childhood Acute Lymphoblastic Leukemia Survivors. Curr Treat Options Oncol. 2016; 17(9): 50, doi: 10.1007/s11864016-0426-0, indexed in Pubmed: 27476159.

11. Stephen MD, Zage PE, Waguespack SG. Gonadotropin-dependent precocious puberty: neoplastic causes and endocrine considerations. Int J Pediatr Endocrinol. 2011; 2011: 184502, doi: 10.1155/2011/184502, indexed in Pubmed: 21603196.

12. Chemaitilly W, Sklar CA. Endocrine complications in long-term survivors of childhood cancers. Endocr Relat Cancer. 2010; 17(3): R141-R159, doi: 10.1677/ERC-10-0002, indexed in Pubmed: 20453080.

13. WallaceW, Thomson AB, Kelsey TW. The radiosensitivity of the human oocyte. Human Reproduction. 2003; 18(1): 117-121, doi: 10.1093/humrep/deg016.

14. Ben-Aharon I, Meizner I, Granot T, et al. Chemotherapy-induced ovarian failure as a prototype for acute vascular toxicity. Oncologist. 2012; 17(11): 1386-1393, doi: 10.1634/theoncologist.2012-0172, indexed in Pubmed: 22956534.

15. Elchuri SV, Patterson BC, Brown M, et al. Low Anti-Müllerian Hormone in Pediatric Cancer Survivors in the Early Years after Gonadotoxic Therapy. J Pediatr Adolesc Gynecol. 2016; 29(4): 393-399, doi: 10.1016/j. jpag.2016.02.009, indexed in Pubmed: 26924632.

16. Lunsford AJ, Whelan K, McCormick K, et al. Antimüllerian hormone as a measure of reproductive function in female childhood cancer survivors. Fertil Steril. 2014; 101(1): 227-231, doi: 10.1016/j.fertnstert.2013.08.052, indexed in Pubmed: 24083876.

17. Gao W, Liang JX, Yan Q. Exposure to radiation therapy is associated with female reproductive health among childhood cancer survivors: a meta-analysis study. J Assist Reprod Genet. 2015; 32(8): 1179-1186, doi: 10.1007/s10815-015-0490-6, indexed in Pubmed: 25940112.

18. Biedka M, Kuźba-Kryszak T, Nowikiewicz T, et al. Fertility impairment in radiotherapy. Contemp Oncol (Pozn). 2016; 20(3): 199-204, doi: 10.5114/wo.2016.57814, indexed in Pubmed: 27647982.

19. Irtan $S$, Orbach $D$, Helfre $S$, et al. Ovarian transposition in prepubescent and adolescent girls with cancer. Lancet Oncol. 2013; 14(13): e601-e608, doi: 10.1016/S1470-2045(13)70288-2, indexed in Pubmed: 24275133.

20. Blumenfeld Z. Chemotherapy and fertility. Best Pract Res Clin Obstet Gynaecol. 2012; 26(3): 379-390, doi: 10.1016/j.bpobgyn.2011.11.008, indexed in Pubmed: 22281514.

21. Lopes F, Smith R, Anderson RA, et al. Docetaxel induces moderate ovarian toxicity in mice, primarily affecting granulosa cells of early growing follicles. Mol Hum Reprod. 2014; 20(10): 948-959, doi: 10.1093/molehr/gau057, indexed in Pubmed: 25080441.

22. Molgaard-Hansen L, Skou AS, Juul A, et al. Nordic Society of Pediatric Hematology and Oncology. Pubertal development and fertility in survivors of childhood acute myeloid leukemia treated with chemotherapy only: a NOPHO-AML study. Pediatr Blood Cancer. 2013; 60(12): 1988-1995, doi: 10.1002/pbc.24715, indexed in Pubmed: 24038890.

23. Dvorak CC, Gracia CR, Sanders JE, et al. NCl, NHLBI/PBMTC first international conference on late effects after pediatric hematopoietic cell transplantation: endocrine challenges-thyroid dysfunction, growth impairment, bone health, \& reproductive risks. Biol Blood Marrow Transplant. 2011; 17(12): 1725-1738, doi: 10.1016/j.bbmt.2011.10.006, indexed in Pubmed: 22005649.

24. Balduzzi A, Dalle JH, Jahnukainen K, et al. Fertility preservation issues in pediatric hematopoietic stem cell transplantation: practical approaches from the consensus of the Pediatric Diseases Working Party of the EBMT and the International BFM Study Group. Bone Marrow Transplant. 2017; 52(10): 1406-1415, doi: 10.1038/bmt.2017.147, indexed in Pubmed: 28737775.

25. Lara R, Carmen C, Sabine S. Fertility considerations and the pediatric oncology patient. Semin Pediatr Surg. 2016; 25(5): 318-322, doi: 10.1053/j. sempedsurg.2016.09.006, indexed in Pubmed: 27955736.

26. Salama $M$, Isachenko $V$, Isachenko $E$, et al. Updates in preserving reproductive potential of prepubertal girls with cancer: Systematic review. Crit Rev Oncol Hematol. 2016; 103: 10-21, doi: 10.1016/j.critrevonc.2016.04.002, indexed in Pubmed: 27184425.

27. Soda I, Ishiyama H, Ono S, et al. Assessment of transposed ovarian movement: how much of a safety margin should be added during pelvic radiotherapy? J Radiat Res. 2015; 56(2): 354-359, doi: 10.1093/jrr/rru116, indexed in Pubmed: 25589505.

28. Blumenfeld Z. How to preserve fertility in young women exposed to chemotherapy? The role of GnRH agonist cotreatment in addition to cryopreservation of embrya, oocytes, or ovaries. Oncologist. 2007; 12(9): 1044-1054, doi: 10.1634/theoncologist.12-9-1044, indexed in Pubmed: 17914074.

29. Estes SJ. Fertility Preservation in Children and Adolescents. Endocrinol Metab Clin North Am. 2015; 44(4): 799-820, doi: 10.1016/j. ecl.2015.07.005, indexed in Pubmed: 26568494.

30. Johnson EK, Finlayson C, Rowell EE, et al. Fertility Preservation for Pediatric Patients: Current State and Future Possibilities. J Urol. 2017; 198(1): 186-194, doi: 10.1016/j.juro.2016.09.159, indexed in Pubmed: 28189577.

31. Noyes N, Porcu E, Borini A. Over 900 oocyte cryopreservation babies born with no apparent increase in congenital anomalies. Reproductive BioMedicine Online. 2009; 18(6): 769-776, doi: 10.1016/s14726483(10)60025-9.

32. Wallace W, Smith A, Kelsey T, et al. Fertility preservation for girls and young women with cancer: population-based validation of criteria for ovarian tissue cryopreservation. The Lancet Oncology. 2014; 15(10): 1129-1136, doi: 10.1016/s1470-2045(14)70334-1.

33. Ladanyi C, Mor A, Christianson MS, et al. Recent advances in the field of ovarian tissue cryopreservation and opportunities for research. J Assist Reprod Genet. 2017; 34(6): 709-722, doi: 10.1007/s10815-017-0899-1, indexed in Pubmed: 28365839.

34. Demeestere $I$, Simon $P$, Dedeken $L$, et al. Live birth after autograft of ovarian tissue cryopreserved during childhood. Hum Reprod. 2015; 30(9): 2107-2109, doi: 10.1093/humrep/dev128, indexed in Pubmed: 26062556.

35. Wallace WH, Kelsey TW, Anderson RA. Fertility preservation in pre-pubertal girls with cancer: the role of ovarian tissue cryopreservation. Fertil Steril. 2016; 105(1): 6-12, doi: 10.1016/j.fertnstert.2015.11.041, indexed in Pubmed: 26674557. 
36. Practice Committee of American Society for Reproductive Medicine. Ovarian tissue cryopreservation: a committee opinion. Fertil Steril. 2014; 101(5): 1237-1243, doi: 10.1016/j.fertnstert.2014.02.052, indexed in Pubmed: 24684955.

37. Kim SS, Lee WS, Chung MiK, et al. Long-term ovarian function and fertility after heterotopic autotransplantation of cryobanked human ovarian tissue: 8-year experience in cancer patients. Fertil Steril. 2009; 91(6): 2349-2354, doi: 10.1016/j.fertnstert.2008.04.019, indexed in Pubmed: 18675964.

38. Stern CJ, Gook D, Hale LG, et al. First reported clinical pregnancy following heterotopic grafting of cryopreserved ovarian tissue in a woman after a bilateral oophorectomy. Hum Reprod. 2013; 28(11): 2996-2999, doi: 10.1093/humrep/det360, indexed in Pubmed: 24014608.

39. Loren AW, Mangu PB, Beck LN, et al. American Society of Clinical Oncology. Fertility preservation for patients with cancer: American Society of Clinical Oncology clinical practice guideline update. J Clin Oncol.
2013; 31(19): 2500-2510, doi: 10.1200/JCO.2013.49.2678, indexed in Pubmed: 23715580

40. Terenziani M, Spinelli M, Jankovic M, et al. PanCare Network. Practices of pediatric oncology and hematology providers regarding fertility issues: a European survey. Pediatr Blood Cancer. 2014; 61(11): 2054-2058, doi: 10.1002/pbc.25163, indexed in Pubmed: 25065353.

41. Diesch T, Rovo A, von der Weid N, et al. Fertility preservation practices in pediatric and adolescent cancer patients undergoing HSCT in Europe: a population-based survey. Bone Marrow Transplant. 2017; 52(7): 1022-1028, doi: 10.1038/bmt.2016.363, indexed in Pubmed: 28112743.

42. Font-Gonzalez A, Mulder RL, Loeffen EAH, et al. PanCareLIFE Consortium. Fertility preservation in children, adolescents, and young adults with cancer: Quality of clinical practice guidelines and variations in recommendations. Cancer. 2016; 122(14):2216-2223, doi: 10.1002/cncr.30047, indexed in Pubmed: 27175973. 